Diabetes, Bluthochdruck und $\mathrm{Co}$.

\title{
Vier Stunden Sitzen sind schon zu viel!
}

Keine gute Nachricht für Büromenschen: Wer täglich vier Stunden oder mehr auf Hocker, Stuhl oder Sessel zubringt, erhöht sein Risiko, einen Diabetes zu entwickeln.

Dass das Sitzen ein unabhängiger Risikofaktor für Diabetes und andere chronische Erkrankungen sein könnte, legt eine aktuelle Auswertung der australischen „45 and Up“-Studie - einer Langzeit-Kohortenstudie über das gesunde Altern - nahe, in der Emma George von der Universität in Sydney und ihre Kollegen untersuchten, welche Lebensstilfaktoren für den im Vergleich zu Frauen durchschnittlich schlechteren Gesundheitszustand von Männern verantwortlich sein könnten.

Insgesamt befragten sie 643.048 Männer zwischen 45 und 64 Jahren zu ihrer körperlichen Verfassung und ihren Lebensge- wohnheiten. Neben Körpergewicht, Rauchverhalten, chronischen Krankheiten und körperlichen Einschränkungen interessierte sie vor allem, wie viele Stunden die Probanden pro Tag sitzen, ob sie Sport treiben und wenn ja, wie intensiv.

\section{Fast die Hälfte}

\section{chronisch krank}

Die Frage nach einer chronischen Krankheit bejahten 41,3\% der Befragten: 5,6\% gaben an, an Krebs erkrankt zu sein bzw. einmal erkrankt gewesen zu sein, $8,6 \%$ berichteten über Herzprobleme, 7,7\% über Diabetes und 31,3\% über hohen Blutdruck. Länger als acht Stunden täglich saß etwa ein Drittel der Befragten $(32,8 \%)$, wobei jedoch $80,9 \%$ der Männer angaben, in der vorangegangenen Woche mehr als 150 Minuten körperlich aktiv gewesen zu sein. Als absolute Bewegungsmuffel bezeichneten sich nur $4,1 \%$.
$71,9 \%$ waren übergewichtig oder fettleibig, 48,1\% körperlich eingeschränkt.

Im Vergleich zu den Männern, die weniger als vier Stunden am Tag auf ihren vier Buchstaben verbrachten, litten Männer mit einer längeren Sitzdauer - unabhängig vom Alter und anderen Faktoren - signifikant häufiger an chronischen Erkrankungen. Die Sitzdauer erhöhte das Risiko jedoch nicht für alle Erkrankungen in gleichem Maße: Unter Berücksichtigung der Kofaktoren wie Alter, BMI, körperliche Aktivität und körperliche Einschränkung stieg zwar mit der Sitzdauer linear das Diabetesrisiko, weniger deutlich fiel jedoch der Zusammenhang bei Krebs, Bluthochdruck und Herzerkrankungen aus.

(dk)

George ES et al, International Journal of Behavioral Nutrition and Physical Activity 2013, 10:20, doi:10.1186/1479-5868-10-20

\section{Schwangeren-Screening}

\section{Wie zuverlässig ist der 50-g-oGTT?}

Der 50-g-Glukosetoleranztest hat den Vorteil, dass die Patientinnen hierzu nicht nüchtern in die Praxis kommen müssen. Doch welchen Einfluss hat der Zeitpunkt der letzten Mahlzeit auf die Aussagekraft des Tests bei Schwangeren?

Dieser Frage gingen taiwanesische Autoren in einer prospektiven Studie nach. Sie werteten die Ergebnisse von 1387 Schwangeren aus, die sich in der 24.-28. SSW einem oralen Glukose-Screening (50-goGTT) unterzogen. Je nachdem, wann die Frauen vor dem Test gegessen hatten, wurden sie in drei Gruppen eingeteilt. Frauen, die länger als zwei Stunden zuvor nichts mehr zu sich genommen hatten, wurden der „Fasting-Gruppe“ zugeordnet, Frauen, die ein bis zwei Stunden zuvor oder weniger als eine Stunde vor dem Test Nahrung aufgenommen hatten, wurden in der „Fed-Gruppe“ zusammenge- fasst. Bei auffälligem 50-g-oGTT wurde ein Bestätigungstest mit $100 \mathrm{~g}$ Glukose angeschlossen.

\section{Das Essen macht den Unterschied}

Bei Frauen mit Glukosetoleranzstörung und einer Fastenzeit von unter einer Stunde lag der mittlere Wert nach $50 \mathrm{~g}$ Glukose signifikant höher als in der Gruppe derer, die mehr als zwei Stunden vor dem Test nichts gegessen hatten (159,5 vs. 153 mg/dl). Keine signifikanten Unterschiede zwischen den Gruppen zeigten sich dagegen bei Frauen mit normaler Glukosetoleranz sowie bei Schwangeren mit GDM.

Nach Überprüfung der auffälligen 50-g-oGTTs mittels 100-g-oGGT zeigte sich ein klarer Zusammenhang zwischen den „Hungerzeiten“ und dem Vorhersagewert des Screening-Tests für den Gestationsdiabetes. Der 50-g-oGTT scheint stark an Aussagekraft zu gewinnen, wenn die Frauen zwei Stunden vor dem Test nüchtern bleiben.

\section{0-g-Screening zu oft falsch positiv} Während die deutsche Mutterschaftsrichtline in ihrer aktualisierten Fassung von 2012 noch den 50-g-oGTT vorsieht und erst bei einer Blutglukose von $\geq 135$ mg/dl den 75-g-Glukosetoleranztest zur Abklärung, empfiehlt die aktuelle Leitlinie von DDG und DGGG bereits seit 2011 ausschließlich den 75-g-oGTT. Die Begründung der Leitlinienautoren: Das 50-g-Screening, bei dem die Patientinnen nicht nüchtern sein müssen, führe zu vielen falsch positiven Ergebnissen und damit zu entsprechend häufigen Nachfolgeuntersuchungen, die Mutter und Kind belasten. 